\title{
INTERPOLAÇÃO DE DADOS METEOROLÓGICOS UTILIZANDO COVARIÁVEIS PARA A REGIÃO METROPOLITANA DE BELO HORIZONTE
}

Luiza Cintra Fernandes ${ }^{1}$

Diego Rodrigues Macedo ${ }^{2}$

\section{Resumo}

A criação de superfícies continuas com informações climáticas espacialmente distribuídas são importantes para diversas aplicações. Há várias metodologias sendo estudados para melhorar a interpolação dos dados meteorológicos escassamente distribuídos, tanto o estudo dos melhores métodos sendo o Thin Plate Spline (TPS) se mostrado mais eficiente para esta aplicação. Como o uso e combinações de covariáveis que melhoram os modelos de interpolação. Assim este trabalho objetivou testar o uso de covariáveis, como imagens do sensor MODIS de LST e cobertura de nuvens, MDE e distância a costa, utilizando TPS, para a região metropolitana de Belo Horizonte. Em geral a acurácia dos modelos foi boa, principalmente a Temperatura que teve RMSE entre 1 e 4 oC. Para as covariáveis utilizadas o efeito na Temperatura e na Precipitação foi marginal, os modelos que não utilizavam covariáveis tiveram erros muito próximos do que os que utilizavam. Já para a velocidade dos ventos o uso de covariável, principalmente a cobertura de nuvens, foi essencial para se ter um modelo com a menor incerteza associada.

Palavras-Chave: Interpolação, TPS,Superfícies Climáticas

\begin{abstract}
Making continuous spatially distributed climates surfaces are important for a range of applications. Many methodologies are being studied to improved sparsely distributed meteorological data interpolation, both the study of the best method, being Thin Plate Spline (TPS) the most used for this application, as the use and combination of covariates that improved the interpolation. This work has the aim to test the use of covariates, as sensor MODIS images, like LST and cloud cover, DEM and distance to coast, using TPS, for the Belo Horizonte metropolitan region. The general model accuracy was good, especially for the temperature, with a RMSE from 1 to $4{ }^{\circ} \mathrm{C}$. The covariates have marginally effects in Temperature and Precipitation estimation, because the models that didn't use any covariates had errors very similar of the models that used them. But for the wind speed the use of covariates, mainly cloud cover, was essential to improve the model.
\end{abstract}

Key words: Interpolation, TPS, ClimateSurafces

${ }_{1}^{1}$ (IGC/UFMG, mestranda em Análise e Modelagem de Sistemas Ambientais) luizacintraf@gmail.com

2 (IGC/UFMG, Prof Adjunto Departamento de Cartografia, Doutor em Ecologia pela UFMG) rodriguesmacedo@gmail.com

Cadernos do Leste

Artigos Científicos

Belo Horizonte, Jan.-Dez. Vol.17, n¹7, 2017 


\section{1- INTRODUÇÃO}

Geralmente dados meteorológicos são escassamente distribuídos e podem ser considerados pontuais. Assim é necessária a criação de superfícies contínuas com informações climáticas espacialmente distribuídas, isso é possível através de métodos de interpolação (ALVAREZ et al, 2014). Com a interpolação são criadas superfícies climáticas utilizadas de ampla aplicação como estudos: ecológicos, hidrológicos, modelagem de fogo e recursos hídricos, entre outros (CHEN ET AL., 2007; TRABUCCO ET AL., 2008; LOARIE ET AL., 2009; GOLDARAG ET AL.,2016).

A Interpolação é definida como a predição dos valores de uma variável primária em pontos dentro da mesma região de locais amostrados (BURROUGH \& MCDONNELL,1998). Existem dois grupos principais de interpolação: os determinísticos e os geoestatísticos. Os métodos determinísticos criam superfícies de dados medidos baseados na extensão de similaridade (inverso da distância ao quadrado) ou no grau de suavização (funções de base radial), sendo métodos que podem forçar resultados a passar pelos valores dos dados ou não. Já métodos geoestatísticos quantificam a correlação espacial entre todos os pontos medidos, levando em conta a configuração espacial dos pontos em torno do local de previsão, utilizando propriedades estatísticas dos dados medidos para produzir mapas em Raster (KAMALI,2015).

Dos métodos mais utilizados para variáveis climáticas estão a Krigagem, métodogeoestatístico e o método de suavização ‘Thin-plateSplines' (TPS), método determinístico. A Krigagem é um método que modela e minimiza a distribuição de erros incluindo dados de auto covariância, já o método TPS é operacionalmente mais simples já que não requer uma estimação a priori da auto-covariância, que geralmente é difícil de ser estimada e validada em locais com poucos pontos. Além disso esse método é computacionalmente mais eficiente permitindo uma detecção robusta de erros nos medidos, o que diminui os erros na predição de superfícies (ARAWOLO, 2017). Arowolo (2017) interpolou dados de 43 estações meteorológicas da Nigéria utilizando longitude, latitude, elevação e distância a linha de costa como variáveis independentes, utilizando os dois métodos e viu que o TPS teve o menor RMSE, indicando como método ótimo.

Além da escolha do melhor método de interpolação muitos estudos estão focandona inclusão de covariáveis e variáveis independentes que ajudam a estimar melhor o modelo. O modelo digital de elevação (MDE) tem sido a covariável mais utilizada na estimação. Com os avanços do sensoriamento remoto, GIS e tecnologia GPS, tem uma gama de variáveis espacialmente explicitas disponíveis para melhor a acurácia da interpolação (Alvarez et al, 2014).Alvarez et al (2014) testou em seu trabalho a combinação de diversas variáveis como MDE, declividade, distância a costa, aspecto, potencia solar, radar e imagens NDVI do MODIS (ModerateResolutionImagingSpectroradiometer) e do AVHRR (AdvancedVery High Cadernos do Leste 
ResolutionRadiometer), verificando que há outras variáveis além do MDE que podem melhorar o modelo. Outros autores como Mildrexleret al 2011 e Jin \& Dickinson, 2010 provaram em seus trabalhos que os dados de temperatura da superfície (LST) do sensor MODIS tem grande correspondência com os dados de temperatura.

Fick\&Hijmans (2017), criaram um banco de dados de informações climáticasinterpoladas no espaço para o mundo inteiro, com uma resolução de $1 \mathrm{~km}$, chamada de WorldClim2. Utilizando TPS eles interpolaram dados de temperatura (máxima, mínima e média), precipitação, radiação solar, pressão atmosférica e velocidade do vento, utilizando covariáveis que incluíam elevação, distancia a costa, LST máxima, mínima e média, e cobertura de nuvens derivadas do MODIS. No fim ele dividiu em 23 regiões cada uma com o modelo que mais se adequou a região.

Interpolar base de dados climáticas em uma escala regional tem a vantagem deincluir informações específicas da região, tendo dados mais claros e tendo melhor controle dos dados, resultando em produtos robustos e confiáveis (CUERVO-ROBAYO, 2013). Assim nesse trabalho o experimento de Fick\&Hijmans (2017) será replicado para uma escala regional para verificar se os melhores modelos convergem com o modelo sugerido a América do Sul. A área de estudo é a Região Metropolitana de Belo Horizonte, utilizando dados de 2014 a 2016, sendo a ideia utilizar esses dados posteriormente como variável de entrada em um modelo de risco de incêndios.

\section{2- METODOLOGIA}

\section{1- Área de estudo}

A Região Metropolitana de Belo Horizonte (RMBH) é composta por 34 municípios,com uma extensão Territorial de $9460 \mathrm{Km} 2$. Trata-se da $3^{\mathrm{a}}$ maior Região Metropolitana do Brasil, apresentando uma população de aproximadamente 5 milhões de habitantes e com um PIB de 56,8 milhões de reais, correspondente a 40\% da economia do estado de Minas Gerais (Agência RMBH, 2016). O clima da região é representado por estações bem demarcadas de chuva e seca, típico de clima tropical. Caracteriza-se por um grande período de seca entre abril e setembro, sendo que junho, julho e agosto apresentam o maior déficit hídrico. 


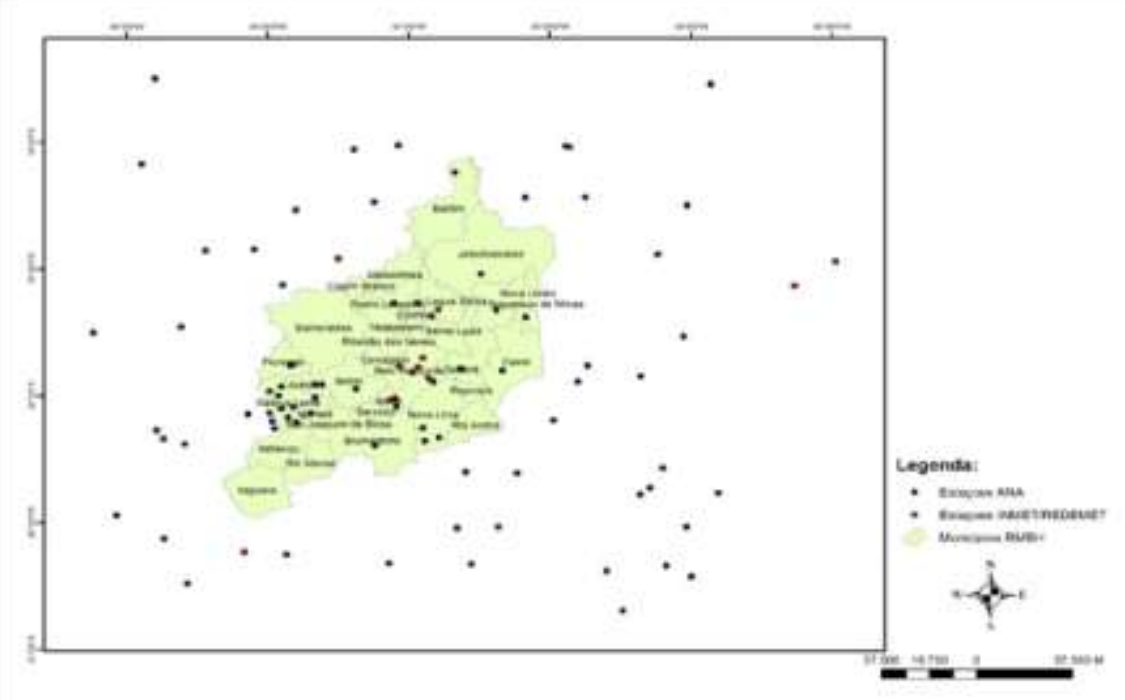

Figura 1 - Área de estudo e estações meteorológicas utilizadas

\section{2- Dados meteorológicos}

Para os dados de precipitação foram selecionadas as estações da Agência Nacionalde Águas (ANA) que continham dados recentes de precipitação e que estavam presentes no entorno e dentro da área de estudo. No total foram 73 estações (figura 1), de onde foi feito o cálculo de média de precipitação anual e a soma da precipitação total por ano. Os dados são disponibilizados pelo site do HIDROWEB (http://hidroweb.ana.gov.br/).

Os dados de temperatura média, temperatura máxima, temperatura mínima, evelocidade dos ventos foram retirados de duas fontes: 6 estações automáticas e 6 convencionais do Instituto Nacional de Meteorologia (INMET), disponíveis no site(http://www.inmet.gov.br/) e 5 estações automáticas da Rede de Meteorologia do Comando da Aeronáutica (REDEMET), totalizando 17 estações na área e entorno (figura 1), onde foi calcula a média anual de cada variável. Os dados de radiação e pressão atmosférica estão apenas disponíveis nos dados do INMET, sendo que o primeiro apenas disponível nos dados das estações automáticas.

\section{3- Covariáveis}

Os dados de satélite utilizados foram os produtos MODIS MOD11A2 e o MOD09GA (disponível em http://ladsweb.nascom.nasa.gov), sendo o primeiro produto que trata da temperatura da superfície da terra (Land SurfaceTemperature - LST), disponibilizados dados da temperatura da superfície de dia (LST-day) e temperatura da superfície a noite (LSTnigth), utilizados como temperatura máxima e mínima, foi feita uma média das duas imagens para encontrar a temperatura média. Estes dados são disponibilizados em escala Kelvin, com um fator de multiplicação de 0,02, resolução temporal de 8 dias e espacial de $1 \mathrm{~km}$, uma média anual dessas imagens foi utilizada. O produto MOD09GA, apresenta em seus subprodutos o estado da Cadernos do Leste 
atmosfera, disponibilizados em forma de bytes, cada valor possui 16 bits, sendo que cada intervalo contem uma informação sobre a condição da atmosfera. Os pixels de 1 a 2 apresentam a condição de nuvem sendo nublado (01), limpo (00) e misto (10). As imagens têm resolução temporal de 1 dia e espacial de $1 \mathrm{~km}$. Para este trabalho foram contabilizados os pixels com a "flag" positiva para nublado, sendo utilizada a porcentagem de dias que apresentaram tempo nublado, calculado para cada ano. A manipulação desses dados foi feita a partir de programas em $\mathrm{R}$.

Para dados do Modelo Digital de Elevação (MDE), foram utilizadas imagem SRTM de resolução de 30 m (disponível em https://earthexplorer.usgs.gov/). Para os dados de distância a costa foi utilizada a linha da costa da América do Sul, e foi calculada a distância euclidiana noArcGis. Os dados do WorldCLim2 (disponível em http://worldclim.org/version2) também foram utilizados como teste para covariável, foram utilizados os dados de 30s. Para utilização como covariáveis e variáveis independentes os dados foram extraídos nas latitudes e longitudes das estações.

\section{4- Interpolação dos Dados e criação das superfícies}

O método de interpolação utilizado foi o Thin Plate Spline (TPS) este métodoconsiste em ajustar uma superfície para os dados com algum erro associado a cada ponto, assim a superfície pode ser mais suave do que se os dados fossem ajustados exatamente. Neste método cada estação é omitida da estimação e o erro médio é encontrado. Isso é repetido para um intervalo de valores de parâmetros suavizadores, então o valor que minimiza o erro é pego para se ter uma suavização ótima. Esse processo é chamado de Validação Cruzada Generalizada (Generalised Cross Validation- GCV). Para isso foi implementado o algoritmo de TPS disponível em linguagem $\mathrm{R}$, na biblioteca "fields". A linguagem R é um ambiente interativo de programação para análise de dados, inferência computacional e visualização. Está em extensão através de funções definidas pelo o usuário e pacotes adicionais. É de disponibilização livre disponível em www.R-project.org e funciona em várias plataformas como UNIX, Windows e MacOS(HORNICk\&LEISH, 2002).

$\mathrm{Na}$ função TPS utilizada no pacote é assumido um modelo aditivo $\mathrm{Y}=\mathrm{f}(\mathrm{X})+$ !, aonde $\mathrm{f}(\mathrm{x})$ é uma superfície dimensional e ! o erro atribuído. Assim o TPS é o resultado da minimização da soma dos resíduos quadrados sujeitos a uma função a certo nível de suavização. A suavização é quantificada pela integral de derivadas quadradas de m-ésima ordem. Por exemplo para função de duas dimensões de ordem 2, a suavização é dada pela equação 1, além de controlar a ordem das derivadas, o valor de $\mathrm{m}$ também determina a base polinomial para ajustar os dados, sendo o grau da polinomial igual a m-1.

$$
I[f(x)]=\iint\left(f_{x x}^{2}+2 f_{x y}^{2}+f_{y y}^{2}\right) d x d y \quad \text { (Eq. 1) }
$$

Cadernos do Leste

Artigos Cientificos

Belo Horizonte, Jan.-Dez. Vol.17, n¹7, 2017 
O modelo tem como entrada as variáveis independentes, que consiste nascoordenadas dos pontos e outras variáveis que se deseje, as variáveis dependentes e eventuais covariáveis. Para interpolar no espaço é utilizada a função "interpolate", que interpola os dados em uma superfície raster. E a função "predict" que prediz os valores para dada coordenadas. A combinação de variáveis utilizadas é apresentada na tabela 1.

Tabela 1 - Modelos de Combinação de Covariáveis Testados

\begin{tabular}{|c|c|}
\hline Modelos & Explicação \\
\hline $\mathbf{f}(\mathbf{x}, \mathbf{y}, \mathbf{z})+\mathbf{s a t}$ & Utilização do MDE como variável independente e dados do MODIS como covariável \\
$\mathbf{f}(\mathbf{x}, \mathbf{y}, \mathbf{z})+\mathbf{c o a s t}$ & Utilização do MDE como variável independente e dados de distância a costa como covariável \\
$\mathbf{f}(\mathbf{x}, \mathbf{y}, \mathbf{z}, \mathbf{s a t})$ & Utilização do MDE e do MODIS como variável independente \\
$\mathbf{f}(\mathbf{x}, \mathbf{y}, \mathbf{z}, \mathbf{c o a s t})$ & Utilização do MDE e de distância a costa como variável independente \\
$\mathbf{f}(\mathbf{x}, \mathbf{y}, \mathbf{z})$ & Utilização do MDE como variável independente \\
$\mathbf{f}(\mathbf{x}, \mathbf{y}, \mathbf{s a t})$ & Utilização dos dados do MODIS como variável independente \\
$\mathbf{f}(\mathbf{x}, \mathbf{y}, \mathbf{s a t})+\mathbf{z}$ & Utilização dos dados do MODIS como variável independente e dados de elevação como variável \\
$\mathbf{f}(\mathbf{x}, \mathbf{y}, \mathbf{z})+\mathbf{c o a s t}+\mathbf{s a t}$ & independente \\
$\mathbf{f}(\mathbf{x}, \mathbf{y})$ & Utilização do MDE como variável independente e dados do MODIS e distância a costa como \\
$\mathbf{f}(\mathbf{x}, \mathbf{y})+\mathbf{s a t}+\mathbf{z}$ & covariáveis \\
$\mathbf{f}(\mathbf{x}, \mathbf{y})+\mathbf{z}$ & Não utilização de covariáveis \\
$\mathbf{f}(\mathbf{x}, \mathbf{y})+\mathbf{w} \mathbf{2}$ & Utilização dos dados do MODIS e distância a costa como covariáveis \\
$\mathbf{f}(\mathbf{x}, \mathbf{y})+\mathbf{s a t}+\mathbf{z}$ & Utilização do MDE como covariável \\
$\mathbf{f}(\mathbf{x}, \mathbf{y})+\mathbf{c o a s t}$ & Utilização dos dados do WorldClim2 como covariável \\
$\mathbf{f}(\mathbf{x , y}, \mathbf{y} \mathbf{c} \mathbf{c})$ & Utilzação do MODIS e MDE como covariável \\
\hline
\end{tabular}

\section{5- Validação do Modelo}

Para validação do modelo foi feita uma validação cruzada estratificada em duasamostras. Para isso a área foi dividida em quatro partes, e em cada área foram selecionados pontos aleatórios, sendo que as duas amostras contêm o mesmo número de estações e as estações das extremidades para garantir o mesmo tamanho da área (figura 2). 


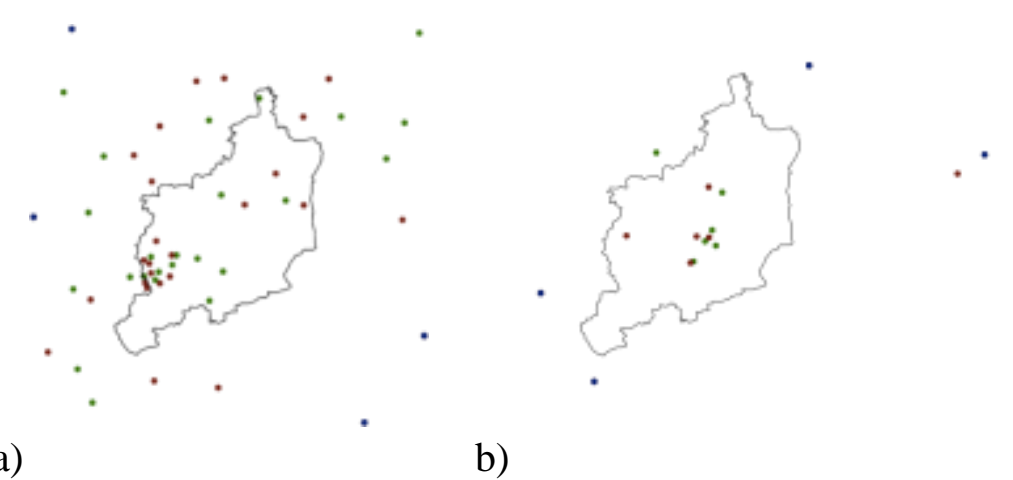

Figura 2- Amostras separadas de treinamento (vermelho) e teste (verde), para precipitação (a) e temperatura e vento (b). As estações em azul, são as estações utilizadas nas duas amostras por serem os extremos

Assim uma amostra é utilizada para treinamento e outra para validação e écalculada a Raiz do Erro Quadrado Médio (Root Mean Square Error - RMSE), Erro Médio Absoluto (MeanAbsoluteError - MAE) e o coeficiente de Pearson (-) entre os valores medidos e preditos. Depois as amostras foram trocadas e calculado os parâmetros novamente. Foram utilizados os dados médios entre os parâmetros das duas amostras para avaliação do melhor modelo.

\section{3- RESULTADOS}

Foram selecionados como melhor modelo aqueles que apresentaram o menor RMSE.

\section{1- Precipitação Média}

No caso da precipitação o melhor modelo para dois anos foi o que utilizou apenas o MDE como variável independente. Mas é possível perceber que o modelo que utiliza as imagens do MODIS como variável independente teve um RMSE bem próximo do outro modelo. Assim os dois modelos poderiam ser utilizados na interpolação dos dados. Também é possível perceber que o modelo que utiliza apenas as coordenadas das estações teve um RMSE baixo, inclusive para o ano de 2015 foi o melhor modelo.

Separou-se os dados em períodos de seca (abril a setembro) e chuva (outubro a março) para saber se há diferenças entre os períodos. Onde foi possível notar que para o período de chuva o modelo utilizando a cobertura de nuvens como variável independente foi o melhor, enquanto que no período de seca ele obteve o mesmo RMSE, do utilizando dados de elevação, com o coeficiente de Pearson maior.

\section{2- Temperatura}

Para análise de temperatura não foi possível calcular todos os modelos devido aalguns produzirem matrizes colineares. Nota-se que o modelo utilizando os dados de satélite como Cadernos do Leste 
variável independente $(\mathrm{f}(\mathrm{x}, \mathrm{y}, \mathrm{sat}))$, o modelo utilizando o MDE como variável independente $(\mathrm{f}(\mathrm{x}, \mathrm{y}, \mathrm{z}))$, e o modelo sem covariável $(\mathrm{f}(\mathrm{x}, \mathrm{y}))$ variaram pouco o seu RMSE para todas as temperaturas. Sendo que para o ano de 2014 o modelo $f(x, y, s a t)$ foi o melhor para temperatura mínima e máxima, enquanto que em 2015 o melhor modelo foi o f(x,y,z) e para 2016 o melhor para temperatura máxima foi $f(x, y)$ e temperatura mínima $f(x, y, s a t)$. Já para a temperatura média em todos os anos os melhores modelos foram $\mathrm{f}(\mathrm{x}, \mathrm{y})$.

Separou-se períodos de chuva e seca para temperatura, mas os resultados não foram muito diferentes. Para a temperatura média, nos dois períodos o melhor modelo foi utilizando os dados do MODIS, já para temperatura mínima ele foi melhor apenas no período de seca, sendo que para o de chuva o melhor foi o utilizando MDE como variável independente. Já para Temperatura máxima no período de chuva o melhor foi o $\mathrm{f}(\mathrm{x}, \mathrm{y}, \mathrm{sat})$, enquanto que para o período de seca foi o sem utilizar covariáveis.

\section{3- Velocidade do vento}

Os dados de vento tiveram uma melhor acurácia em seus modelos quandoadicionada a variável de cobertura de nuvem como variável independente para os anos de 2014 e 2015, apenas o ano de 2016 que o modelo com elevação teve uma melhor performance. Já quando se separou os dados por períodos nota-se que no período de chuvas o melhor modelo foi o que considera todas as variáveis, tendo o MDE como independente e a distância a costa e cobertura de nuvens como covariável. Enquanto no período de seca o modelo utilizando os dados de satélite foi melhor.

\section{4- Geração de superfícies}

Verificado os melhores modelos estes foram utilizados na confecção dassuperfícies climáticas dos três anos, os resultados do ano de 2014 pode ser visto na figura 3.

Estas foram comparadas com os resultados do WorldCLim2.

\section{5- Discussão}

A acurácia geral dos modelos foi boa, já que ficou entre 2 e 4oC para os melhoresmodelos de temperatura máxima e mínima, e entre 1 e 2 oC para os melhores modelos de temperatura média. No caso da precipitação a acurácia ficou entre 12-22 $\mathrm{mm}$. Quanto aos ventos a acurácia não foi muito boa, ficando entre 3-4 m/s, mas isso foi devido a pequena rede de estações e a grande variabilidade entre os dados, que variavam de $12 \mathrm{~m} / \mathrm{s}$ a $0.95 \mathrm{~m} / \mathrm{s}$.

Os resultados encontrados se diferenciaram dos modelos para a América do Sul encontrados por Fick\&Hijmans (2017), isso porque o clima tem uma grande variabilidade e se comporta diferente em cada região. É importante salientar que no estudo de Fick\&Hijmans (2017) eles fizeram a interpolação para o mundo todo, separando em apenas 23 regiões. Sendo que o Cadernos do Leste 
modelo que abrange o Brasil, é o mesmo para toda a América do Sul. Os dados do WorldClim 2, produzidos por Fick\&Hijmans (2017), foram testados como covariável e em todos os casos apresentaram baixa acurácia e correlação. Fazendo a comparação visual entre eles (figura 3), notase grandes diferenças entre eles, tanto nos valores máximos e mínimos, quanto a distribuição no espaço, isso porque esse produto utiliza média de 30 anos, e fez um modelo para a América do Sul inteira, perdendo efeitos locais e temporais.

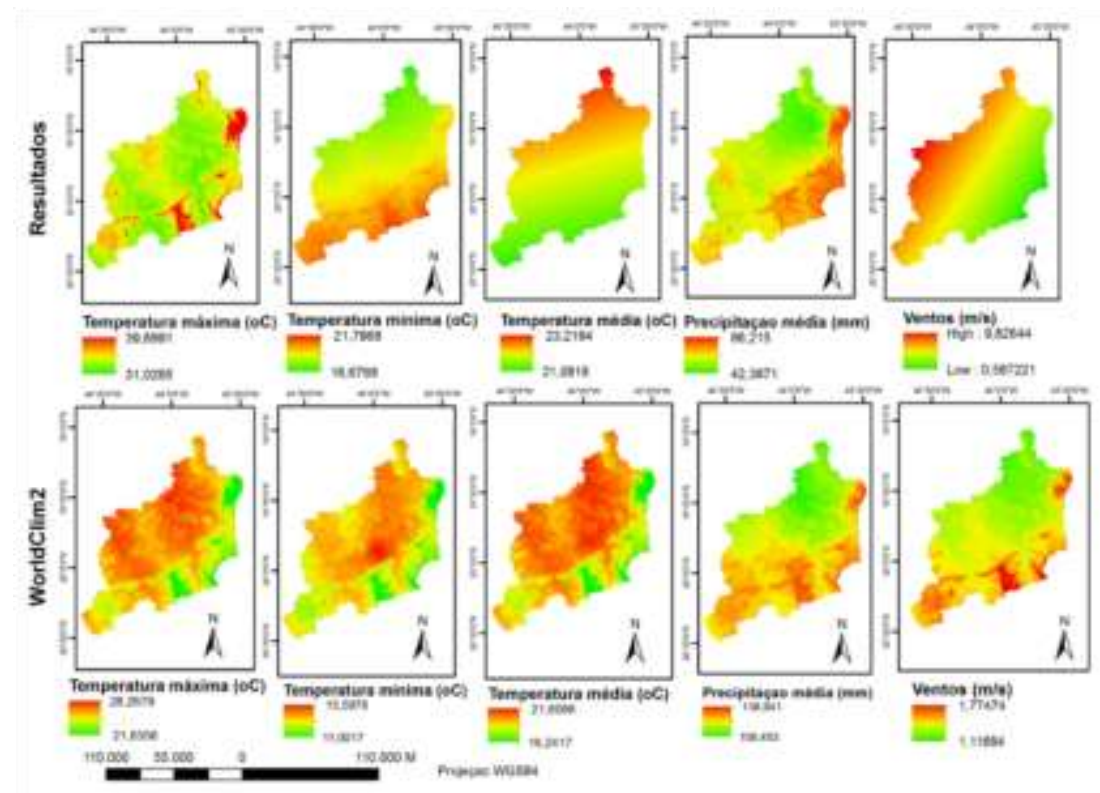

Figura 3 - Resultados das superfícies climáticas para o ano de 2014 e comparação com os dados do WorldCLim2

A adição de outras variáveis como variável independente ou covariável nãomelhorou muito a estimação para os dados de precipitação e temperatura. Os dados de distância a costa chegaram a piorar bastante o modelo, já os dados de elevação e cobertura de nuvens tiveram efeitos marginais na estimação da temperatura, assim como os dados de temperatura da superfície para a estimação das temperaturas.

Quanto a estimação de velocidade do vento a adição de cobertura de nuvens e elevação como variável independente foram essenciais para o modelo, já que tiveram um erro bem menor do que o modelo bruto. As duas variáveis tiveram efeitos parecidos nos modelos. Quando separado por épocas o melhor modelo é quando são adicionadas todas as variáveis, cobertura de nuvens, elevação e distância a costa, isso porque a velocidade dos ventos pode estar associada a vários fatores. Nota-se um aumento na correlação dos dados de satélite com dados de ventos do que na estimação de outras variáveis.

Não foi possível fazer o estudo com as variáveis de radiação solar e pressãoatmosférica porque as estações que continham esses dados eram escassas, assim não era possível separá-las em Cadernos do Leste 
duas amostras para validação. Demonstrando assim a dificuldade de se fazer interpolações climáticas em regiões menores, já que a densidade de estações e disponibilização dos dados é baixa. Por isso muitos estudos são feitos para regiões maiores, o que acaba por formar modelos de interpolação que não agregam efeitos locais.

Alvarez et al (2014) fez uma comparação entre o uso de diversas covariáveis paramelhorar a interpolação de dados de temperatura e precipitação de sua área, que também de dimensões regionais, com $1710000 \mathrm{~km} 2$. O que ele verificou em sua pesquisa é que para precipitação o uso de radar para precipitação foi o que teve o melhor modelo e para temperatura o uso de MDE. Mas também verifica que a combinação de variáveis pode piorar a acurácia do modelo ao invés de melhorar os modelos, pois adicionam maiores incertezas. Em geral a acurácia do seu modelo não foi muito boa com RMSE de $18 \mathrm{oC}$ para as temperaturas e $148 \mathrm{~mm}$ para a precipitação. Ele também verifica que fazendo a interpolação entre os meses do ano separadamente, a acurácia do modelo aumenta. Assim talvez para áreas menores o uso de covariáveis não seja efetivo.

Jin \& Dickinson (2010) em seu trabalho verificaram a correspondência entre o LSTe a temperatura do ar. Em seu estudo eles concluem que o parâmetro da LST é fisicamente diferente da temperatura do ar, pois o primeiro depende de propriedades da superfície e interações na atmosfera. Eles também estudam diferentes escalas temporais e espaciais, e o que eles apontam é que para estudos de escalas globais e de longo termo, a temperatura da superfície pode trazer benefícios, como no estudo de mudanças climáticas por exemplo, mas para escalas locais e regionais essa pode não ser a melhor alternativa. Mildrexler (2011), fez uma comparação entre dados de temperatura do ar provenientes de estações meteorológicas do mundo inteiro com os dados de LST máximo, e verificaram como uma boa alternativa para verificar mudanças climáticas globais, além de mostrar alta relação entre dados de LST e tipos de cobertura da Terra.

Em comparação com estes estudos a utilização dessas covariáveis não ser efetivasem áreas menores, pode ser devido as características dos produtos, que interagem com outros parâmetros, ou devido a resolução espacial e temporal. É interessante se testar covariáveis com uma resolução espacial melhor, como imagens Landsat e dados LASER.

Por outro lado, Henglet al. (2010) utilizou Krigagem com regressão para fazer mapas de temperatura diários em 159 estações na Croácia, utilizando como covariável distancia a costa, elevação, insolação e imagens do LST do MODIS. Onde verificou que adicionar as imagens de LST melhorou a detecção de mudanças locais. Conseguindo uma acurácia global de 91\%. Kilibardaet al. (2014) utilizou o mesmo método, mas para predição global de temperaturas para o ano de 2011, e também verificou que imagens LST do MODIS obtiveram bons resultados, com RMSE de 2oC para áreas de densidade maior de redes e de 4 oC para áreas de densidade menor.

Cadernos do Leste

Artigos Cientificos

Belo Horizonte, Jan.-Dez. Vol.17, n¹7, 2017 


\section{4- CONCLUSÃO}

O modelo de interpolação de dados pode variar de uma região a outra, por isso aimportância de se ter o estudo dos melhores métodos de interpolação e como fazer para melhorálo. A adição de covariáveis e variáveis independentes pode sim melhorar os modelos de interpolação, sendo importante saber qual a que tem melhor correlação ao fenômeno e qual se aplica melhor a área de estudo.

Neste estudo as variáveis adicionadas foram efetivas na interpolação de dados dedireção do vento, mas tiveram efeitos marginais para estimar precipitação e temperatura. Sugere-se utilizar no futuro outras variáveis que se associem melhor a área e que tenham melhor resolução, como imagens das bandas termais do Landsat e dados LASER.

Foi utilizada a metodologia de interpolação do TPS por ser a mais utilizada pelosautores na interpolação de superfícies climáticas. Mas estudos recentes têm utilizados outras técnicas como a Krigagem com regressão que tem obtido resultados melhores, assim em trabalhos futuros sugerese a utilização de outros métodos de interpolação para melhorar a acurácia do modelo.

\section{5- MATERIAL DE APOIO}

Tabela S1- Resultados da validação cruzada para cada elemento climático e modelo

\section{REFERÊNCIAS BIBLIOGRÁFICAS}

AGÊNCIA RMBH. RMBH e Colar Metropolitano. Disponível em: $<$ http://www.agenciarmbh.mg.gov.br/institucional/rmbh-e-colar-metropolitano/>. Acessoem: 20 de outubro de 2016.

ALVAREZ, O., GUO, Q., KLINGER, R. C., LI, W. AND DOHERTY, P. Comparison of elevation and remote sensing derived products as auxiliary data for climate surface interpolation. International Journal of Climatology, v.34, n. 17, p. 2258-2268. Junho de 2014. doi:10.1002/joc.3835

AROWOLO, A. O., BHOWMIK, A. K., QI, W. AND DENG, X. Comparison of spatial interpolation techniques to generate high-resolution climate surfaces for Nigeria. International Journal of Climatology, v.37, n. S1, p.179-192, Agostos de 2017. doi:10.1002/joc.4990

CHEN, H. GUO, S. XU, C-Y. SINGH, VP. Historical temporal trends of hydro-climatic variables and runoff response to climate variability and their relevance in water resource management in the Hanjiang basin. JournalofHydrology. v. 344, n. 3-4, p. 171-184. Outubro de 2007.

CUERVO-ROBAYO, Angela P. et al. An update of high-resolution monthly climate surfaces for Mexico. International Journal of Climatology, v. 34, n. 7, p. 2427-2437, Junho de 2014. 
FICK, S. E. AND HIJMANS, R. J. WorldClim 2: new 1-km spatial resolution climate surfaces for global land areas. InternationalJournalofClimatology, v. 37, n. 12, p. 4302-4315. Outubro de 2017. doi:10.1002/joc.5086

GOLDARAG, Y. J. MOHAMMADZADEH, A. ARDAKANI, A. S. Fire Risk Assessment Using Neural Network and Logistic Regression.Journal of the Indian Society of Remote Sensing, v. 44, n. 6, p. 885-894.Dezembro de 2016.

HORNIK, K. LEISCH, F. Vienna and R: Love, marriage and the future, in: R. Dutter (Ed.), Festschrift50 JahreÖsterreichischeStatistischeGesellschaft, ÖsterreichischeStatistischeGesellschaft 19512001, Austrian Statical Society, pp. 61-70, 2002.

JIN M, DICKINSON RE. Land surface skin temperature climatology: benefitting from the strengths of satellite observations. Environmental Research Letters. V. 5, n. 4, p. 44004. Novembro de 2010.

KAMALI, M. I. et al. The determination of reference evapotranspiration for spatial distribution mapping using geostatistics. Water Resources Management, v. 29, n. 11, p. 3929-3940, setembro de 2015.

LOARIE, S.R. DUFFY, P.B. HAMILTON, H. ASNER, G.P. FIELD, C.B. ACKERLY, D.D.The velocity of climate change.Nature.v.462, n. 7276, p. 1052-1111.Dezembro de 2014.

MILDREXLER, D. J., M. ZHAO, AND S. W. RUNNING (2011), A global comparison between station air temperatures and MODIS land surface temperatures reveals the cooling role of forests. Journal of Geophysical Research. v. 116, n. G03025. Setembro de 2011.doi:10.1029/2010JG001486.

BURROUGH, P.A. MCDONNELL, R.A. Principles of Geographical Information Systems. Oxford: Oxford University Press,1998.

TRABUCCO A, ZOMER RJ, BOSSIO DA, VAN STRAATEN O, VERCHOT LV. Climate change mitigation through afforestation/reforestation: a global analysis of hydrologic impacts with four case studies. Agriculture, ecosystems\&environment. v. 126,n. 1-2, p. 81-97.Junho de 2008. 